\title{
Effect of Ring Strain on the Charge Transport of a Robust Norbornadiene- Quadricyclane-Based Molecular Photoswitch
}

Tebikachew, Behabitu E. ; Li, Haipeng B.; Pirrotta, Alessandro; Börjesson, Karl; Solomon, Gemma C.; Hihath, Joshua ; Moth-Poulsen, Kasper

Published in:

The Journal of Physical Chemistry Part C

DOI:

10.1021/acs.jpcc.7b00319

Publication date:

2017

Document version

Publisher's PDF, also known as Version of record

Document license:

CC BY

Citation for published version (APA):

Tebikachew, B. E., Li, H. B., Pirrotta, A., Börjesson, K., Solomon, G. C., Hihath, J., \& Moth-Poulsen, K. (2017).

Effect of Ring Strain on the Charge Transport of a Robust Norbornadiene-Quadricyclane-Based Molecular Photoswitch. The Journal of Physical Chemistry Part C, 121(13), 7094-7100.

https://doi.org/10.1021/acs.jpcc.7b00319 


\title{
Effect of Ring Strain on the Charge Transport of a Robust Norbornadiene-Quadricyclane-Based Molecular Photoswitch
}

\author{
Behabitu E. Tebikachew, ${ }^{\dagger, \perp}$ Haipeng B. Li, ${ }^{\ddagger}, \perp$ Alessandro Pirrotta, ${ }^{\S}$ (@) Karl Börjesson, ${ }^{\prime \prime}$ \\ Gemma C. Solomon, ${ }^{*}, \odot$ Joshua Hihath, ${ }^{*},+$ and Kasper Moth-Poulsen ${ }^{*}, \oplus$ \\ ${ }^{\dagger}$ Department of Chemistry and Chemical Engineering, Chalmers University of Technology, 41296 Gothenburg, Sweden \\ ${ }^{\ddagger}$ Department of Electrical and Computer Engineering, University of California Davis, Davis, California 95616, United States \\ ${ }^{\S}$ Nano-Science Center and Department of Chemistry, University of Copenhagen, 2100, Copenhagen $\varnothing$, Denmark \\ "Department of Chemistry and Molecular Biology, University of Gothenburg, 41296 Gothenburg, Sweden
}

\section{Supporting Information}

ABSTRACT: Integrating functional molecules into single-molecule devices is a key step toward the realization of future computing machines based on the smallest possible components. In this context, photoswitching molecules that can make a transition between high and low conductivity in response to light are attractive candidates. Here we present the synthesis and conductance properties of a new type of robust molecular photothermal switch based on the norbornadiene (NB)quadricyclane (QC) system. The transport through the molecule in the ON state is dominated by a pathway through the $\pi$ conjugated system, which is no longer available when the system is switched to the OFF state. Interestingly, in the OFF state we find that the same pathway contributes only $12 \%$ to the transport properties. We attribute this observation to the strained tetrahedral geometry of the QC. These results challenge the prevailing assumption that current will simply flow through the shortest through-bond path in a molecule.

\section{INTRODUCTION}

Integrating functional molecules into single-molecule devices is a key step toward the realization of future computing machines based on the smallest possible component, single molecules. ${ }^{1-4}$ In this context, molecular switches that can make a transition between high and low conductivity in response to external stimuli have attracted significant attention since they can add a unique electronic function to molecular-scale devices and at the same time provide insight into the charge transport mechanism at the nanoscale. ${ }^{5-10}$

Photoswitching molecules, molecules that undergoes a welldefined structural isomerization upon photoexcitation, are appealing candidates for single-molecule electronics. ${ }^{3,11}$ There are several examples of photoswitching molecules. From a charge transport perspective diarylethenes ${ }^{12-15}$ and azobenzenes $5,16,17$ have been the two major molecular platforms rigorously studied through various experimental approaches in the past decade. Recently, Jia et al. demonstrated a robust diarylethene-based device with remarkable stability and bidirectional in situ switching with graphene electrodes. ${ }^{15}$ Other photoswitchable molecules with more complicated structures have also been investigated. ${ }^{7,19}$ Here, we designed and synthesized a norbornadiene-based photoswitch (NB-1) with embedded oligo(phenylene ethynylene) arms end-capped with thioacetate groups to anchor it to gold electrodes (Scheme 1).

Norbornadiene is a bicyclic hydrocarbon that undergoes photoisomerization to the metastable quadricyclane form upon light stimulation. The quadricyclane form can relax back to

Scheme 1. Photochemical Isomerization of the Norbornadiene Form (NB-1) to the Quadricyclane Form (QC-2) and the Thermal Relaxation
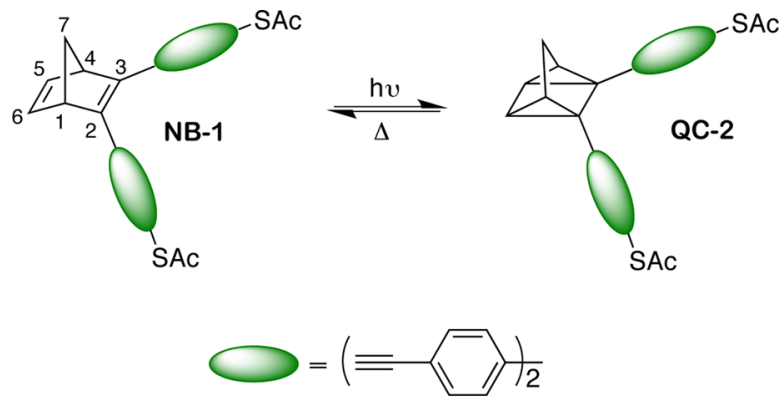

Received: January 11, 2017

Revised: $\quad$ March 1, 2017

Published: March 6, 2017 
norbornadiene by thermal activation. Hence, it is one of the few examples of a T-type negative photochrome along with dimethyldihydropyrene. ${ }^{18}$ The photoswitching properties of norbornadiene have been demonstrated and utilized to study intramolecular energy transfer in solution. In 1994, Bonfantini et al. proposed norbornadiene as a potential candidate system for molecular electronics. ${ }^{20}$ The prospect of using norbornadiene in electronic applications has also been discussed by Löfås et al. ${ }^{21}$ Yet, to the best of our knowledge, there are no examples of experimental conductance studies regarding the evaluation and use of the norbornadiene system as a photoswitch in molecular electronics. Hence, NB-1 is characterized in solution, revealing robust photoswtiching properties, and its transport properties are investigated in STM-break junction measurements. Furthermore, the transport properties of the system are analyzed in depth using DFT modeling and local current analysis.

Electron transport through NB-1 is dominated by the $\pi$ system. However, upon photoexcitation the central unit undergoes an intramolecular $[2+2]$ cycloaddition, rehybridizing the orbitals from $\mathrm{sp}^{2}$ to $\mathrm{sp}^{3}$, to give the photoisomer, $\mathrm{QC}$ 2 (Scheme 1). ${ }^{22}$ In this state, the conductance must be dominated by a pathway involving the $\sigma$ system, as no fully conjugated path remains. As a result, our measurements yield a larger on/off ratio than expected from breaking a single $\pi$-bond in the transport pathway. The DFT calculations show that the conductance in QC-2 is characterized by a path through the longer cyclobutane three $\sigma$-bonds over the shorter single $\sigma$ bond path, and the resulting switching ratio is in good agreement with the experimental results. This work brings more insight into the effect of quantum interference on charge transport in strained ring structures and suggests new candidates and perspectives on engineering molecular-level photoswitches.

\section{RESULTS AND DISCUSSION}

The photoswitching molecule (NB-1) was synthesized through Sonogashira $^{23}$ cross-coupling reaction starting from 2,3dibromonorbornadiene. Full synthetic details and characterization are presented in the SI. The photoisomerization process was examined in solution using NMR and UV-vis spectroscopy. The proton NMR for the photoisomerization of NB-1 to QC-2 showed an alkenyl proton for NB-1, whereas in QC-2 the alkenyl protons disappeared and new alkyl protons emerge (SI: Figure S3). UV-vis absorption spectroscopy of NB-1 was carried out in toluene, and the onset of absorption wavelength was found to be around $460 \mathrm{~nm}$ (SI: Figure S4(a)), which is significantly red-shifted from unsubstituted norbornadiene. This is attributed to the extended $\pi$-conjugation in NB-1, which enabled the photoisomerization with visible light. Irradiating a toluene solution of NB-1 with visible light resulted in its photoisomerization to QC-2 with isosbestic points in the absorption spectra indicating a clean photoconversion (Figure $1(\mathrm{a}))$.

In order to examine the robustness and fatigue resistance of the photoswitch, a toluene solution of NB-1 $(\sim 44 \mu \mathrm{M})$ was irradiated with a $405 \mathrm{~nm}$ laser diode at $50{ }^{\circ} \mathrm{C}$ for $2 \mathrm{~min}$ in order to fully convert the NB-1 to QC-2. The ensuing QC-2 was allowed to relax for $28 \mathrm{~min}$ at this temperature prior to the next irradiation cycle. The conversion was continuously probed at $430 \mathrm{~nm}$. Under nitrogen atmosphere, ${ }^{24}$ the compound showed no sign of degradation even after well over 100 switching cycles (Figure 1(c)). In air, more than 100 switching cycles were
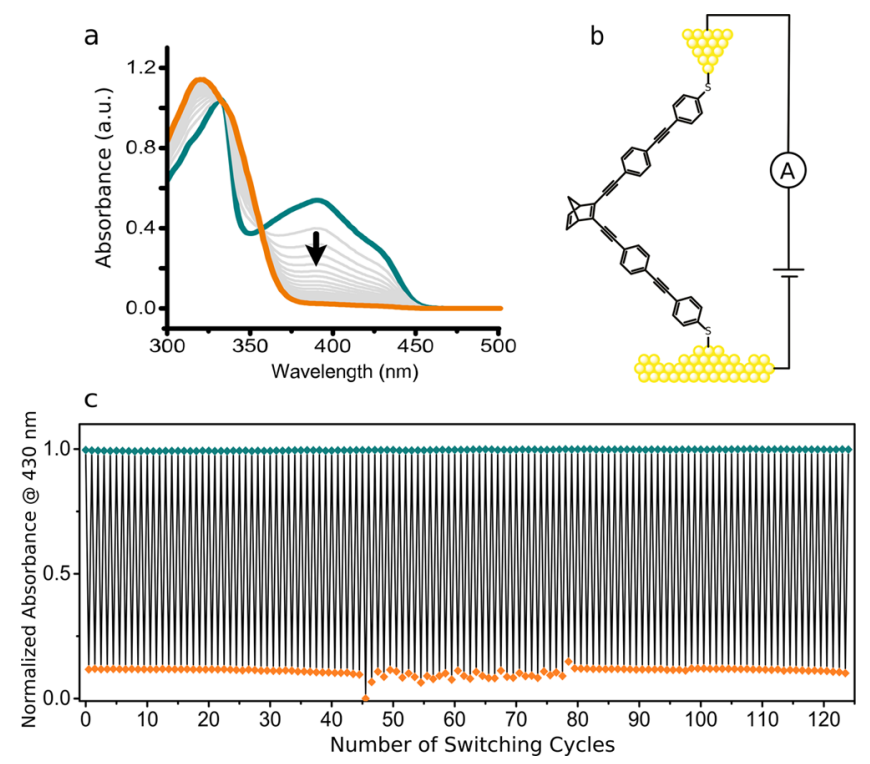

Figure 1. (a) Photoisomerization of NB-1 (dark cyan) to QC-2 (orange) indicated by the black arrow followed by UV/vis spectroscopy. The photoisomerization was performed using a $405 \mathrm{~nm}$ laser diode, and the sample was exposed to the irradiation every $10 \mathrm{~s}$ until the complete conversion of NB-1 through the gray plots to QC-2 was reached. (b) Sketch of device geometry under test in the STM break junction measurements. (c) No sign of degradation was observed in the accelerated stability test of the NB-1 (dark cyan maxima)/QC-2 (orange minima) system at elevated temperature $\left(50{ }^{\circ} \mathrm{C}\right)$ sealed under nitrogen atmosphere; 124 photothermal switching cycles are shown.

performed with only $16 \%$ loss (SI: Figure S4(f)), corresponding to $99.8 \%$ yield per conversion cycle. This experiment reveals the robustness of the photoswitch, even at elevated temperatures.

The thermal relaxation of QC-2 to NB-1 over time was further investigated at different temperatures and found to follow first-order kinetics. The rate constants for the thermal switching reaction at a range of temperatures were extracted from the exponential fitting of the relaxation of QC-2 to NB-1. Rate constants obtained at four different temperatures (SI: Figure S4(b)) were fitted to the Arrhenius equation yielding a straight line (SI: Figure S4(c)). From the plot, the activation energy $\left(E_{\mathrm{a}}\right)$ as well as the pre-exponential factor (A) were determined for the thermal back conversion. The extracted values are summarized in Table 1.

Having demonstrated that the molecule effectively switches from the $\pi$-conjugated NB-1 state to the QC-2 state and vice versa in solution, we turn our attention to the charge transport properties of the system. STM-break junction (STM-BJ) experiments have been proven to be a robust method to obtain conductance values of single molecular junctions. ${ }^{26-33}$ This technique was used to examine the change in conductance at the single-molecule level between molecules NB-1 and QC-2 in order to gain insight into the details of the charge transport mechanism $^{34}$ (Figure 2). In STM-BJ a bias is applied between the tip and substrate, and the current is measured continuously as the tip is being retracted. Steps on the decaying conductance traces appear whenever molecules are bound between the two electrodes. With thousands of conductance vs distance curves collected, the most probable conductance value of the singlemolecule junction can be determined by statistical analysis. 
Table 1. Photochemical and Thermodynamic Parameters for NB-1 and QC-2 ${ }^{a, b}$

$\begin{array}{cccccc}\left(\lambda_{\mathrm{NB}-1 \max } \varepsilon\right)\left(\mathrm{nm}, \mathrm{M}^{-1} \mathrm{~cm}^{-1}\right) & \left(\lambda_{\mathrm{QC}-2 \max \varepsilon}\right)\left(\mathrm{nm}, \mathrm{M}^{-1} \mathrm{~cm}^{-1}\right) & E_{\mathrm{a}}\left(\mathrm{kJ} \mathrm{mol}^{-1}\right) & A\left(\mathrm{~s}^{-1}\right) & t_{1 / 2,25^{\circ} \mathrm{C}}(\mathrm{min}) & \phi^{*} \\ \left(332,8.3 \times 10^{4}\right),\left(391,4.3 \times 10^{4}\right) & \left(320,9.1 \times 10^{4}\right) & 100.7 & 1.6 \times 10^{14} & 78.6\end{array}$

${ }^{a}$ Summary of calculated Arrhenius parameters for the thermal relaxation and photoisomerization quantum yield of QC-2 to NB-1. ${ }^{b} \varepsilon$, molar extinction coefficient of NB-1 (two maxima) and QC-2 (one maximum); $E_{a}$ activation energy for the thermal relaxation of QC-2 to NB-1 derived from the Arrhenius equation; $A$, pre-exponential factor; $t_{1 / 2} 25^{\circ} \mathrm{C}$, half-life time of the thermal relaxation of QC-2 at $25^{\circ} \mathrm{C}$; $\phi^{*}$, photoisomerization quantum yield ${ }^{25}$ of NB-1 to QC-2 obtained from the average of three separate measurements.
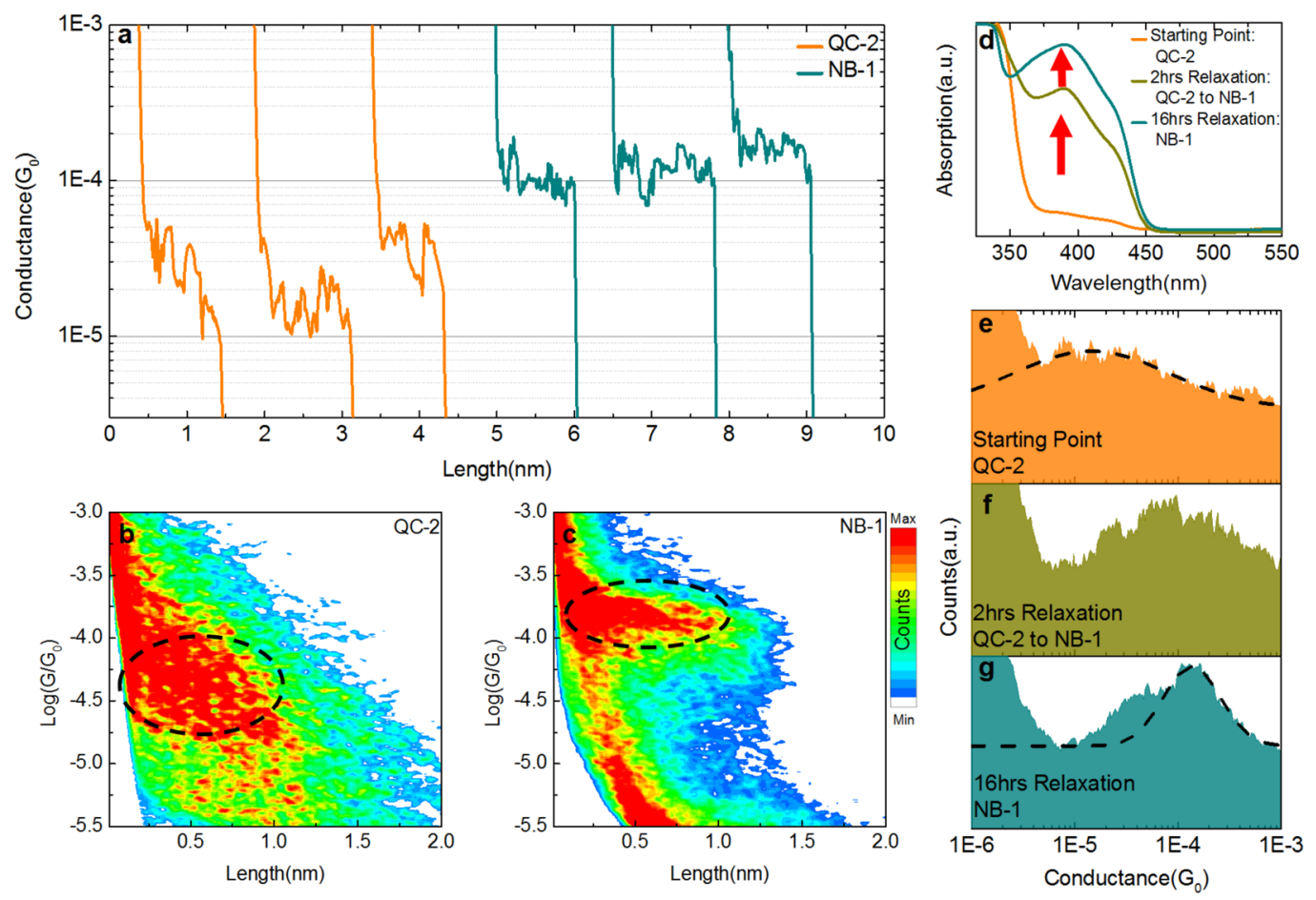

Figure 2. (a) Conductance vs length traces of the NB-1 state and the QC-2 state. Traces are displaced horizontally for clarity. (b) and (c) 2D histogram for QC-2 and NB-1 demonstrating a large change in the distribution of conductance values for the two states. (d) UV-vis spectrum for the relaxation of QC-2 to NB-1 in 16 h. (e)-(g) Conductance histograms of QC-2 before relaxation (e), after $2 \mathrm{~h}$ of relaxation (f), and after $16 \mathrm{~h}$ relaxation $(\mathrm{g})$. All the experiments were carried out at room temperature $\sim 23{ }^{\circ} \mathrm{C}$.

To measure the conductance value of the norbornadienebased photoswitching molecule at both NB-1 and QC-2 states, NB-1 was dissolved in mesitylene. The solution was then added to the STM cell $(\sim 1 \mu \mathrm{M}$ in mesitylene), and a $50 \mathrm{mV}$ bias was applied between the gold tip and the substrate, and the tapping process as described above was initiated with a tip speed of $\sim 80$ $\mathrm{nm} / \mathrm{s}$. For the QC-2 state, a UV-LED (centered at $405 \mathrm{~nm}$ ) was employed to switch the molecule from NB-1 to QC-2 in solution phase, and the absorption spectrum was measured to verify the full conversion from NB-1 to QC-2. The STM-BJ conductance measurement for $\mathbf{Q C}-2$ was performed under continuous irradiation of UV-light to hinder the relaxation from QC-2 back to NB-1, and data collection was finished within 30 min after adding QC-2 to the cell.

Figure 2a shows individual decay curves for both QC-2 (orange traces) and NB-1 (dark cyan traces). It is observed that the steps of QC-2 are at lower conductance values compared to NB-1. By automatically selecting decay curves with clear steps, 2D histograms are constructed for QC-2 and NB-1 in Figure 2(b),(c). NB-1 yields a conductance value on the order of $10^{-4}$ $G_{0}$, while QC-2 has a lower value on the order of $10^{-5} G_{0}$. Five sets of STM-BJ conductance measurements have been performed for both NB-1 and QC-2 states. Each measurement is based on the newly prepared substrate, solution, and gold tip.
The data are plotted in the 1D conductance histogram and fitted with a Gaussian distribution. The average conductance values each from the five experimental data sets are summarized in Table 2. The conductance values indicate that the change in molecular configuration directly leads to a conductance switch of molecular junction, with an ON/OFF ratio of 6.6.

Table 2. Conductance Measurement Results for NB-1 and QC-2 Forms

$\begin{array}{ccc}\text { NB-1 conductance } & \text { QC-2 conductance } & \text { conductance ratio } \\ 1.2 \pm 0.1 \times 10^{-4} G_{0} & 1.9 \pm 0.8 \times 10^{-5} G_{0} & 6.6\end{array}$

To further confirm the correlation between states of the molecule and the junction conductance, in situ conductance measurements were performed in parallel with UV-vis absorption spectroscopy as the molecules relaxed from QC-2 to NB-1 (Figure 2(d)-(g)). A solution of QC-2 was initially added to the STM cell $(\sim 1 \mu \mathrm{M}$ in mesitylene), and three consecutive in situ conductance measurements were performed: immediately after molecular addition, $2 \mathrm{~h}$ later, and $16 \mathrm{~h}$ later. The system was maintained in a dark environment at room temperature throughout the experiment. Figure 2(e) shows a peak in the conductance histogram centered at $\sim 1.9 \times 10^{-5} G_{0}$ 

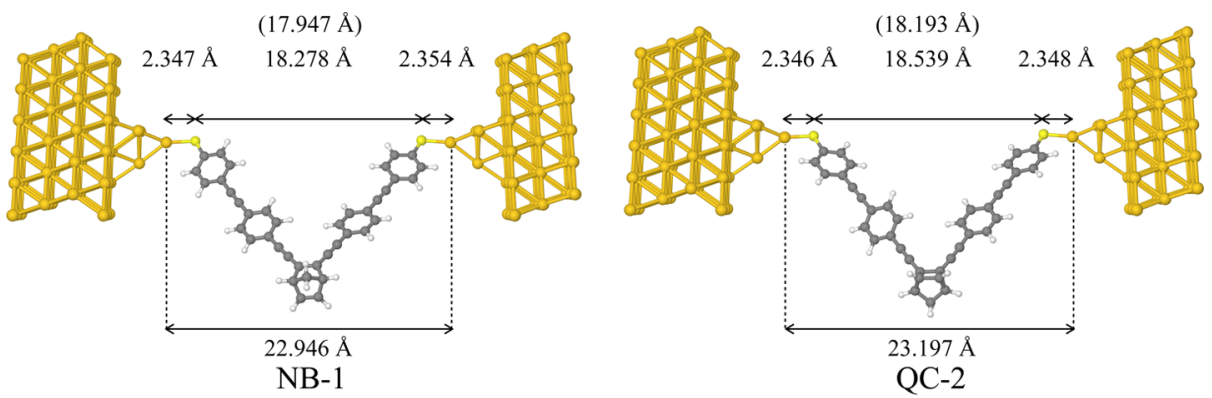

Figure 3. Optimized structure of the device region (scattering region) for NB-1 and QC-2 molecular junctions. The lengths in brackets correspond to the $\mathrm{S}-\mathrm{S}$ distance of the gas-phase relaxed structures.

immediately following the addition of the QC-2 solution. Figure 2(f) shows the conductance histogram obtained after $2 \mathrm{~h}$ of relaxation and shows an evolution of the conductance distribution. The diminished low conductance peak and increased counts at high conductance range indicate the relaxation process from QC-2 to NB-1 is occurring. Finally, Figure $2(\mathrm{~g})$ was obtained after $16 \mathrm{~h}$ of relaxation. A more distinct peak with a value around $1.2 \times 10^{-4} G_{0}$ is observed, indicating a complete relaxation. A control experiment with absorption spectrum measured in the solution phase was performed at the same time, and as demonstrated in Figure 2d, QC-2 underwent essentially complete relaxation process to NB-1 in 16 h, which is $99.99 \%$ back conversion calculated based on the values presented in Table 1.

The in situ relaxation STM break junction experiment suggests that the direct cause of the conductance change is the isomerization of the photoswitching molecule. We also tested switching NB-1 molecules to the QC-2 state by irradiating UV light onto the gold surface after molecules bound to gold, but no obvious conductance change was observed. We attribute this phenomenon to the rapid quenching of the excited states due to electronic coupling ${ }^{35}$ once the molecule is coupled to the gold surface. Perhaps, this effect can be alleviated by inserting a saturated spacer between the photoactive unit and the electrode ${ }^{15}$ or by using a different anchoring group. ${ }^{36}$ Detailed data for the STM-BJ measurements can be found in the SI Section III.

One of the advantages of the NB-1/QC-2 system is that the bonds that are broken and formed upon switching along the main conduction path undergo no skeletal rearrangement. ${ }^{37}$ Hence, we expect the end-to-end length to remain largely unaffected. This will also yield junctions that are mechanically stable during the switching event. However, the limited structural change presents a potential problem for maximizing the conductance ON/OFF ratio.

It is possible to make an estimate of the conductance change upon switching when only a single bond changes from conjugated to saturated. By assuming that current only flows through the shortest through-bond path, knowledge of the characteristic conductance decay with length for conjugated and saturated systems allows us to estimate the difference between the two systems (full details in SI, section IV). This calculation yields a first estimate for the ON/OFF ratio of about 2 , a very modest switching ratio. This calculation takes a simple view of conduction through the molecule and neglects through-space interactions or any contributions from longer pathways through the cyclic unit. While these assumptions might seem reasonable as a starting point, this raises a common challenge when trying to rationalize molecular electronics measurements: whether molecules can be considered as simply a number of bonds/resistors in series.

To understand the ON/OFF ratio observed, we simulated the transport properties for NB-1 and QC-2 using quantum chemical modeling as shown in Figure 3. Full details of the simulation method are given in the SI (Section IV).

The calculated transmission for the two molecular junctions using DFTB+ is shown in Figure 4(a). The transmission through molecules terminated with thiols is usually dominated by a transmission resonance associated with the highest occupied molecular orbital (HOMO), whose energy lies close to the Fermi energy of the gold electrodes. ${ }^{38,39}$

Our findings are in accordance with this: for both systems, the transmission peak close to the HOMO energy lies near the Fermi energy, and its tail is responsible for the transmission at the Fermi energy. Due to the smaller energy gap between occupied and virtual orbitals of $\pi$-conjugated molecules, the decay of the tail of the HOMO peak in NB-1 is slower than in QC-2 where the gap is larger. This results in a higher transmission for NB-1 at the Fermi energy. The calculated transmission ratio between NB-1 and QC-2 at the Fermi energy is 12 . The trends observed in the transmission were also reproduced in DFT transmission calculations as shown in the SI (Figure S7).

While the transmission calculations confirm that we expect an ON/OFF ratio of significantly more than 2 , the total transmission alone does not give us any insight into why this is the case. To examine this point, we probe the transport pathways through NB-1 and QC-2 using local current analysis, ${ }^{40}$ the results of which are shown in Figure 4(b).

Local currents of NB-1 show that the current goes through the $\mathrm{C}-\mathrm{C}$ double bond as expected, with no evidence of the current being dominated by anything other than the shortest through-bond path. However, the situation with QC-2 is somewhat more complicated. The simple picture of the current pathway had one double bond being replaced by one single bond, but this is not the dominant pathway we observe. In fact, the double bond is "replaced" by three $\sigma$ bonds, with $88 \%$ of the current flowing out through the longer cyclobutane unit of QC-2 and only $12 \%$ taking the shortest through-bond path. Interestingly, if we perform the same back-of-the-envelope calculation assuming that we replace one conjugated bond with three saturated bonds we obtain an ON/OFF ratio of 28 .

Clearly, the assumption that the current will always favor the shortest through-bond path breaks down in QC-2. Previous theoretical work on fully conjugated molecules has shown that the current may take a longer path when the shortest path is dominated by quantum interference. ${ }^{41}$ The geometry of the shortest path in QC-2 is indeed very close to a cis defect, which 


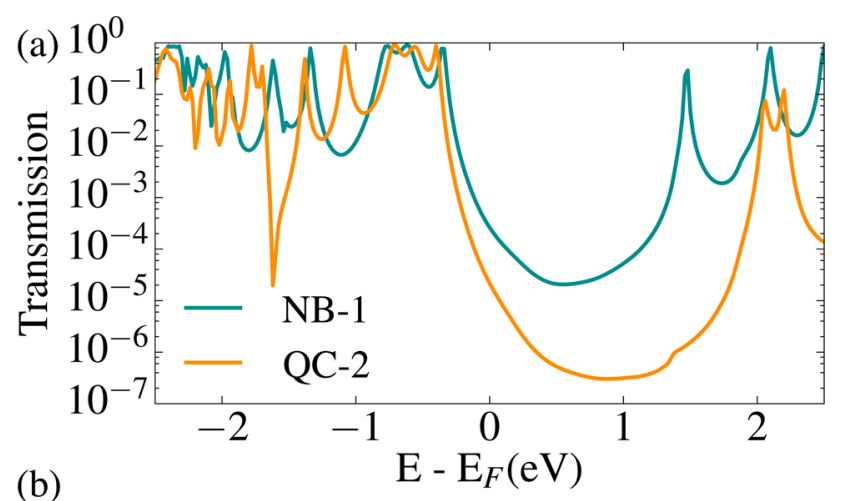

(b)

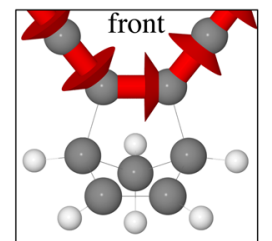

NB-1

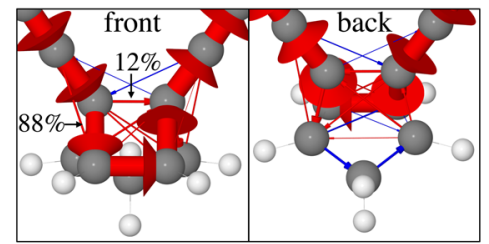

QC-2

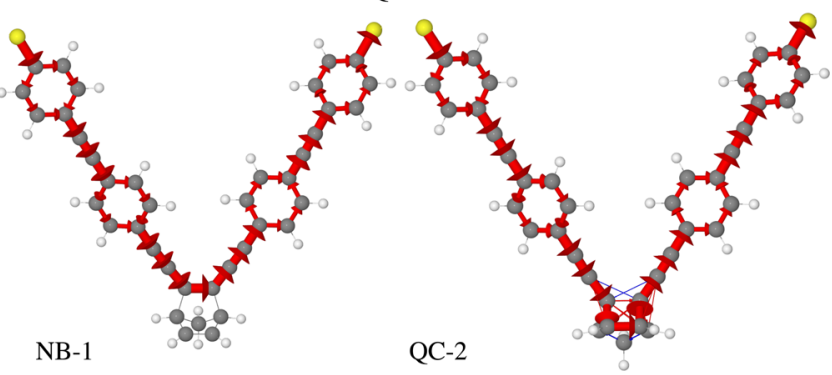

Figure 4. (a) Transmission calculated with DFTB+ and the auorg parameters for the molecules NB-1 and QC-2 sandwiched between the gold electrodes. (b) Local currents calculated with DFTB+ at the Fermi level energy with a symmetric bias of $0.1 \mathrm{eV}$. The red arrows indicate a positive contribution to the current density, while the (small) blue arrows indicate a negative contribution (vide zoomed out front and back insets Figure 4(b)). The photochemically active region affects the electron transport properties: a fully conjugated path in NB$\mathbf{1}$ is replaced by a saturated ring, which reduces the electron transmission. Moreover, the saturated region in QC-2 perturbs the current pathway: only $12 \%$ of the current goes through the shortest $\sigma$ path, while the $88 \%$ goes through three $\sigma$ bonds.

has been shown to reduce the current ${ }^{42}$ and exhibit interference effects. 38,40

When looking for ring-current reversals as the signature of interference, ${ }^{40}$ we see some evidence of interference in QC-2 (Figures S8 and S9); however, the picture is not so definitive. What is clear is that some local current elements reverse direction, and the current through the shortest pathway goes through a minimum (significantly below the $12 \%$ seen at the Fermi energy) around the same energy as the transmission minimum. What this picture does not tell us is why the longer path is favored, as this is also a $\sigma$ pathway with a dihedral close to zero.

We can probe this question by computing the local currents through a series of molecules with and without cyclopropanestrained carbons to determine which geometric features control the balance of current through the long and short paths (Figure 5 ). These molecular structures have been generated by simply removing atoms from the $\mathbf{Q C}-\mathbf{2}$ relaxed structure and replacing them with hydrogen atoms that are then relaxed while keeping all parts of the original QC-2 structure fixed. We find that the

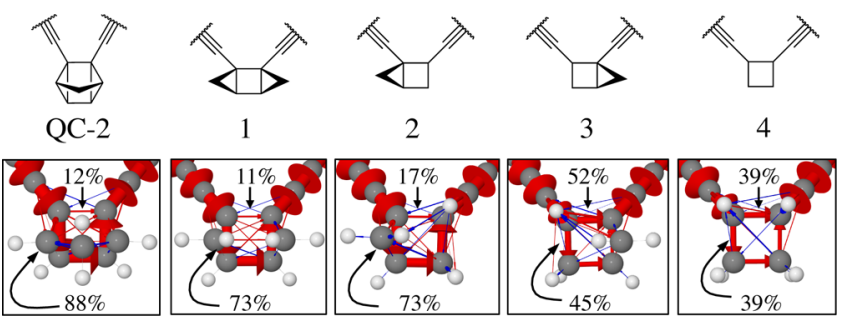

Figure 5. DFTB+ local currents for a series of modified QC-2 structures. This analysis shows that the strained geometry of the cyclopropane rings significantly affects the local features of the current. Specifically, the strained geometry changes the balance between the short and long path.

symmetry of the carbons connecting the quadricyclane unit to the $\pi$-conjugated arms changes the balance between the current densities going through the long and the short path. When the carbons assume a strained $\mathrm{sp}^{3}$ symmetry (Figure 5 structure 1 ) the longer path is preferred. When the cyclopropane rings are removed, we find a balance close to $50 / 50$ between the long and short path (Figure 5 structure 4 ).

While theory and experiment both demonstrate that the ON/OFF ratio should be higher than 2, the agreement between the two is certainly not quantitative. As always with these types of transport calculations, band line-up between the electrode Fermi energy and the molecular energy levels is far from perfect. ${ }^{43}$ Both DFTB+ and DFT calculations suffer from this problem, and it is clear from the transmission that the ON/ OFF ratio predicted is sensitive to the position of the Fermi level. Beyond this issue, it is also known that treating the effective single-particle Hamiltonian from a DFT calculation as a true single-particle Hamiltonian for the purposes of a transport calculation may not always be a good approximation. ${ }^{38}$

Chemically, we are also left with the question of whether the partitioning of the current through a polycyclic system into interatomic "bond current" contributions effectively describes the transport. Saturated systems are known to exhibit both surface and volume delocalization, ${ }^{44}$ which suggests the current might also flow through the space within the QC-2 core. It remains a question for future work as to when a "bond current" type representation is accurate, as opposed to a real-space local current representation. ${ }^{45,46}$

\section{CONCLUSION}

In conclusion, we have designed, synthesized, and characterized a thiol end-capped norbornadiene-based photoswitch, NB-1. The NB-1 photoswitch is found to have high fatigue resistance and excellent cyclability. Under nitrogen no sign of degradation was observed during well over 100 switching cycles at $50{ }^{\circ} \mathrm{C}$. An STM-BJ conductance study revealed two conductance states, high $\left(1.2 \pm 0.1 \times 10^{-4} G_{0}\right)$ and low $\left(1.9 \pm 0.8 \times 10^{-5}\right.$ $\left.G_{0}\right)$ conductances. While the switching ratio of the NB-1/QC-2 photoswitch pair is somewhat lower than some other wellstudied systems, ${ }^{15,17}$ it discloses a significantly different approach for charge transport mechanisms.

Through DFT calculations, we have shown that the NB-1/ QC-2 photoswitch system effectively modulates the conductance through a junction, not only by breaking the conjugation but also by forcing the current to take a longer three-bond path in QC-2 through the saturated cyclobutane moiety. This result challenges the assumption that current will 
simply flow through the shortest through-bond path and reminds us that the intricacies of quantum systems should not be ignored; i.e., it can be problematic to consider molecules and especially cage-like structures such as the QC-2 core as a sum of bonds. This is not to say that molecules generally cannot be considered as a sum of their parts; in fact, this has been shown to be an effective picture in many cases. ${ }^{47}$ It simply raises the question of how far one can break down the system, and clearly there are functional groups that need to be considered as single units, rather than a sum of bonds.

In general, despite the need to improve the quantum yield for real-world applications as is the case with other photoswitch systems, ${ }^{12}$ this study opens up a new perspective on tuning molecular conductance: using interference effects to effectively shuttle current between different parts of a molecule.

\section{ASSOCIATED CONTENT}

\section{S Supporting Information}

The Supporting Information is available free of charge on the ACS Publications website at DOI: 10.1021/acs.jpcc.7b00319.

Detailed synthetic procedure, NMR spectra, absorption spectra, electrochemcial measurement, STM-BJ measurements, and the supporting quantum chemical calculations (PDF)

\section{AUTHOR INFORMATION}

\section{Corresponding Authors}

*E-mail: gsolomon@chem.ku.dk.

*E-mail: jhihath@ucdavis.edu.

*E-mail: mkasper@chalmers.se.

\section{ORCID 1}

Alessandro Pirrotta: 0000-0001-5487-1299

Gemma C. Solomon: 0000-0002-2018-1529

Kasper Moth-Poulsen: 0000-0003-4018-4927

\section{Author Contributions}

${ }^{\perp}$ B.E.T. and H.B.L. contributed equally. The manuscript was written through contributions of all authors. All authors have given approval to the final version of the manuscript.

\section{Notes}

The authors declare no competing financial interest.

\section{ACKNOWLEDGMENTS}

BET and KMP acknowledge funding from European Research Council (ERC-StG \#337221, SIMONE). AP thanks Gabriele Penazzi for help with the transport calculations using DFTB+. $\mathrm{AP}$ and GCS received funding from the Danish Council for Independent Research I Natural Sciences and the Carlsberg Foundation. JH acknowledges support from the U.S. Office of Naval Research and the National Science Foundation. KB acknowledges funding from the Swedish Foundation for Strategic Research.

\section{REFERENCES}

(1) Aviram, A.; Ratner, M. A. Molecular Rectifiers. Chem. Phys. Lett. 1974, 29, 277-283.

(2) Haddon, R. C.; Lamola, A. A. The Molecular Electronic Device and the Biochip Computer: Present Status. Proc. Natl. Acad. Sci. U. S. A. 1985, 82, 1874-1878.

(3) Xiang, D.; Wang, X.; Jia, C.; Lee, T.; Guo, X. Molecular-Scale Electronics: From Concept to Function. Chem. Rev. 2016, 116, 43184440.
(4) Sun, L.; Diaz-Fernandez, Y. a; Gschneidtner, T. a; Westerlund, F.; Lara-Avila, S.; Moth-Poulsen, K. Single-Molecule Electronics: From Chemical Design to Functional Devices. Chem. Soc. Rev. 2014, 43, 7378-7411.

(5) Zhang, C.; Du, M. H.; Cheng, H. P.; Zhang, X. G.; Roitberg, A. E.; Krause, J. L. Coherent Electron Transport through an Azobenzene Molecule: A Light-Driven Molecular Switch. Phys. Rev. Lett. 2004, 92, 158301.

(6) Taherinia, D.; Frisbie, C. D. Photoswitchable Hopping Transport in Molecular Wires $4 \mathrm{Nm}$ in Length. J. Phys. Chem. C 2016, 120, $6442-6449$.

(7) Broman, S. L.; Lara-Avila, S.; Thisted, C. L.; Bond, A. D.; Kubatkin, S.; Danilov, A.; Nielsen, M. B. Dihydroazulene Photoswitch Operating in Sequential Tunneling Regime: Synthesis and SingleMolecule Junction Studies. Adv. Funct. Mater. 2012, 22, 4249-4258.

(8) Tao, N. J. Electron Transport in Molecular Junctions. Nat. Nanotechnol. 2006, 1, 173-181.

(9) Guédon, C. M.; Valkenier, H.; Markussen, T.; Thygesen, K. S.; Hummelen, J. C.; van der Molen, S. J. Observation of Quantum Interference in Molecular Charge Transport. Nat. Nanotechnol. 2012, $7,305-309$.

(10) Lindsay, S. M.; Ratner, M. A. Molecular Transport Junctions: Clearing Mists. Adv. Mater. 2007, 19, 23-31.

(11) Zhang, J. L.; Zhong, J. Q.; Lin, J. D.; Hu, W. P.; Wu, K.; Xu, G. Q.; Wee, A. T. S.; Chen, W. Towards Single Molecule Switches. Chem. Soc. Rev. 2015, 44, 2998-3022.

(12) Irie, M. Diarylethenes for Memories and Switches. Chem. Rev. 2000, 100, 1685-1716.

(13) Matsuda, K.; Irie, M. Diarylethene as a Photoswitching Unit. J. Photochem. Photobiol., C 2004, 5, 169-182.

(14) Kim, Y.; Hellmuth, T. J.; Sysoiev, D.; Pauly, F.; Pietsch, T.; Wolf, J.; Erbe, A.; Huhn, T.; Groth, U.; Steiner, U. E.; et al. Charge Transport Characteristics of Diarylethene Photoswitching SingleMolecule Junctions. Nano Lett. 2012, 12, 3736-3742.

(15) Jia, C.; Migliore, A.; Xin, N.; Huang, S.; Wang, J.; Yang, Q.; Wang, S.; Chen, H.; Wang, D.; Feng, B.; et al. Covalently Bonded Single-Molecule Junctions with Stable and Reversible Photoswitched Conductivity. Science 2016, 352, 1443-1445.

(16) Kumar, A. S.; Ye, T.; Takami, T.; Yu, B. C.; Flatt, A. K.; Tour, J. M.; Weiss, P. S. Reversible Photo-Switching of Single Azobenzene Molecules in Controlled Nanoscale Environments. Nano Lett. 2008, 8, 1644-1648.

(17) Mativetsky, J. M.; Pace, G.; Elbing, M.; Rampi, M. A.; Mayor, M.; Samorì, P. Azobenzenes as Light-Controlled Molecular Electronic Switches in Nanoscale Metal-molecule-metal Junctions. J. Am. Chem. Soc. 2008, 130, 9192-9193.

(18) Roldan, D.; Kaliginedi, V.; Cobo, S.; Kolivoska, V.; Bucher, C.; Hong, W.; Royal, G.; Wandlowski, T. Charge Transport in Photoswitchable Dimethyldihydropyrene-Type Single-Molecule Junctions. J. Am. Chem. Soc. 2013, 135, 5974-5977.

(19) Battacharyya, S.; Kibel, A.; Kodis, G.; Liddell, P. A.; Gervaldo, M.; Gust, D.; Lindsay, S. Optical Modulation of Molecular Conductance. Nano Lett. 2011, 11, 2709-2714.

(20) Bonfantini, E. E.; Officer, D. L. The Synthesis of Norbornadienes Conjugatively Linked to Tetraphenylporphyrin and Anthracene: Towards a Norbornadiene-Derived Molecular Electronic Device. J. Chem. Soc., Chem. Commun. 1994, 1445-1446.

(21) Löfås, H.; Jahn, B. O.; Wärnå, J.; Emanuelsson, R.; Ahuja, R.; Grigoriev, A.; Ottosson, H. A Computational Study of Potential Molecular Switches That Exploit Baird's Rule on Excited-State Aromaticity and Antiaromaticity. Faraday Discuss. 2014, 174, 105124.

(22) Cristol, S.; Snell, R. Bridged Polycyclic Compounds. VI. The Photoisomerization of Bicyclo Hepta-2, 5-Diene-2, 3-Dicarboxylic Acid to quadricyclo[2,2,1,0] Heptane-2, 3-Dicarboxylic Acid. J. Am. Chem. Soc. 1958, 80, 1950-1952.

(23) Chinchilla, R; Nájera, C. Recent Advances in Sonogashira Reactions. Chem. Soc. Rev. 2011, 40, 5084-5121. 
(24) Dreos, A.; Borjesson, K.; Wang, Z.; Roffey, A.; Norwood, Z.; Kushnir, D.; Moth-Poulsen, K. Exploring the Potential of a Hybrid Device Combining Solar Water Heating and Molecular Solar Thermal Energy Storage. Energy Environ. Sci. 2017, 10, 728-734.

(25) Stranius, K.; Börjesson, K. Determining the Photoisomerization Quantum Yield of Photoswitchable Molecules in Solution and in the Solid State. Sci. Rep. 2017, 7, 41145.

(26) Xiao, X.; Xu, B.; Tao, N. J. Measurement of Single Molecule Conductance: Benzenedithiol and Benzenedimethanethiol. Nano Lett. 2004, 4, 267-271.

(27) Venkataraman, L.; Klare, J. E.; Nuckolls, C.; Hybertsen, M. S.; Steigerwald, M. L. Dependence of Single-Molecule Junction Conductance on Molecular Conformation. Nature 2006, 442, 904907.

(28) Aradhya, S. V.; Meisner, J. S.; Krikorian, M.; Ahn, S.; Parameswaran, R.; Steigerwald, M. L.; Nuckolls, C.; Venkataraman, L. Dissecting Contact Mechanics from Quantum Interference in Single-Molecule Junctions of Stilbene Derivatives. Nano Lett. 2012, 12, 1643-1647.

(29) Agraï, N.; Yeyati, A. L.; van Ruitenbeek, J. M. Quantum Properties of Atomic-Sized Conductors. Phys. Rep. 2003, 377, 81-279.

(30) Gittins, D. I.; Bethell, D.; Schiffrin, D. J.; Nichols, R. J. A Nanometre-Scale Electronic Switch Consisting of a Metal Cluster and Redox-Addressable Groups. Nature 2000, 408, 67-69.

(31) Cui, X. D.; Primak, A.; Zarate, X.; Tomfohr, J.; Sankey, O. F.; Moore, A. L.; Moore, T. A.; Gust, D.; Harris, G.; Lindsay, S. M. Reproducible Measurement of Single-Molecule Conductivity. Science 2001, 294, 571-574.

(32) Rascón-Ramos, H.; Artés, J. M.; Li, Y.; Hihath, J. Binding Configurations and Intramolecular Strain in Single-Molecule Devices. Nat. Mater. 2015, 14, 517-522.

(33) Aragonès, A. C.; Aravena, D.; Cerdá, J. I.; Acís-Castillo, Z.; Li, H.; Real, J. A.; Sanz, F.; Hihath, J.; Ruiz, E.; Díez-Pérez, I. Large Conductance Switching in a Single-Molecule Device through Room Temperature Spin-Dependent Transport. Nano Lett. 2016, 16, 218226.

(34) Xu, B.; Tao, N. J. Measurement of Single-Molecule Resistance by Repeated Formation of Molecular Junctions. Science 2003, 301, 1221-1223.

(35) Dulić, D.; van der Molen, S. J.; Kudernac, T.; Jonkman, H. T.; de Jong, J. J. D.; Bowden, T. N.; van Esch, J.; Feringa, B. L.; van Wees, B. J. One-Way Optoelectronic Switching of Photochromic Molecules on Gold. Phys. Rev. Lett. 2003, 91, 207402.

(36) Su, T. A.; Neupane, M.; Steigerwald, M. L.; Venkataraman, L.; Nuckolls, C. Chemical Principles of Single-Molecule Electronics. Nat. Rev. Mater. 2016, 1, 16002.

(37) Dauben, W. G.; Cargill, R. L. Photochemical transformationsVIII The Isomerization of bicyclo[2.2.1] heptadiene to quadricyclo[2.2.1.0.0]heptane (Quadricyclane). Tetrahedron 1961, 15, 197-201.

(38) Tan, A.; Balachandran, J.; Sadat, S.; Gavini, V.; Dunietz, B. D.; Jang, S.-Y.; Reddy, P. Effect of Length and Contact Chemistry on the Electronic Structure and Thermoelectric Properties of Molecular Junctions. J. Am. Chem. Soc. 2011, 133, 8838-8841.

(39) Malen, J. A.; Doak, P.; Baheti, K.; Tilley, T. D.; Segalman, R. A.; Majumdar, A. Identifying the Length Dependence of Orbital Alignment and Contact Coupling in Molecular Heterojunctions. Nano Lett. 2009, 9, 1164-1169.

(40) Solomon, G. C.; Herrmann, C.; Hansen, T.; Mujica, V.; Ratner, M. a. Exploring Local Currents in Molecular Junctions. Nat. Chem. 2010, 2, 223-228.

(41) Tsuji, Y.; Movassagh, R.; Datta, S.; Hoffmann, R. Exponential Attenuation of through-Bond Transmission in a Polyene: Theory and Potential Realizations. ACS Nano 2015, 9, 11109-11120.

(42) Li, C.; Pobelov, I.; Wandlowski, T.; Bagrets, A.; Arnold, A.; Evers, F. Charge Transport in Single AulalkanedithiollAu Junctions: Coordination Geometries and Conformational Degrees of Freedom. J. Am. Chem. Soc. 2008, 130, 318-326.
(43) Strange, M.; Rostgaard, C.; Häkkinen, H.; Thygesen, K. S. SelfConsistent GW Calculations of Electronic Transport in Thiol- and Amine-Linked Molecular Junctions. Phys. Rev. B: Condens. Matter Mater. Phys. 2011, 83, 1-12.

(44) Cremer, D. Pros and Cons of $\sigma$-Aromaticity. Tetrahedron 1988, 44, 7427-7454.

(45) Xue, Y.; Ratner, M. A. Local Field Effects in Current Transport through Molecular Electronic Devices: Current Density Profiles and Local Nonequilibrium Electron Distributions. Phys. Rev. B: Condens. Matter Mater. Phys. 2004, 70, 81404.

(46) Walz, M.; Bagrets, A.; Evers, F. Local Current Density Calculations for Molecular Films from Ab Initio. J. Chem. Theory Comput. 2015, 11, 5161-5176.

(47) Manrique, D. Z.; Al-Galiby, Q.; Hong, W.; Lambert, C. J. A New Approach to Materials Discovery for Electronic and Thermoelectric Properties of Single-Molecule Junctions. Nano Lett. 2016, 16, 13081316. 